\section{The virus of acute diarrhoea}

from Anthea Thornton

and Arie J. Zuckerman

ACUTE infectious gastroenteritis has long been one of the commonest causes of childhood illness throughout the world. In England and Wales (1961) acute diarrhoea accounted for $6 \%$ of deaths in the first year of childhood. But in technically underdeveloped countries, where malnutrition and other debilitating diseases are undoubtedly important contributing factors, deaths from acute diarrhoeal disease in early life are of such magnitude as to be a leading cause of mortality. Probably no more than an eighth of the infants and children of the world are free from appreciable risk of a lethal diarrhoea. Some episodes of gastroenteritis are clearly associated with certain bacterial pathogens; in at least $65 \%$ of cases, however, such agents cannot be isolated. Viruses of various type have occasionally been isolated but in most cases their pathogenic role has remained unestablished.

Recently a new candidate virus, originally classified as a member of the orbivirus group, was seen in epithelial cells of duodenal mucosa from children with acute non-bacterial gastroenteritis (Bishop et al., Lancet, ii, 1281-1283; 1973) and subsequently in negatively stained faecal extracts from such children (Flewett et al., Lancet, ii, 1497; 1973). Morphologically similar particles have since been detected in duodenal fluids and faecal extracts by numerous other workers. Throughout the world, these viruses have now been reported in 396 out of a total of $827(48 \%)$ of children with acute enteritis, and in only two of 357 children without infection. They have rarely been detected in children over the age of six, and are more often encountered in winter. There is now convincing evidence that the new virus is an aetiological agent in acute childhood gastroenteritis. The virus infects epithelial cells only during the symptomatic stage of the illness and its presence coincides with histological abnormalities and depressed duodenal mucosa disaccharidase levels. Seroconversion has been demonstrated in some children and oral transmission of infection has been achieved with one adult volunteer.

The virus differs from both the reovirus and orbivirus group in serology, morphology and polypeptide composition. The virions consist of a core about $38 \mathrm{~nm}$ in diameter, bounded by a membrane from which short cylindrical capsomeres radiate outwards like the spokes of a wheel. An outer layer of capsid subunits seems to be attached to the tops of the inner ones; they are

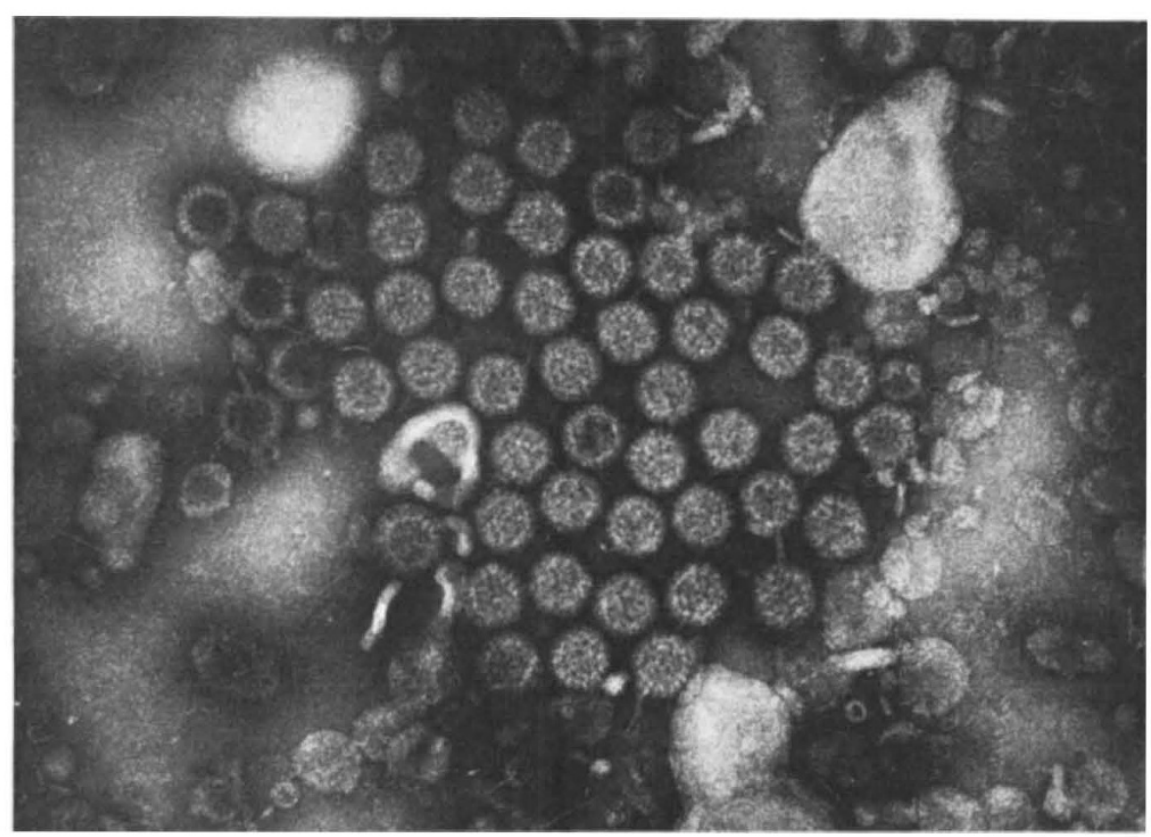

The characteristic morphological appearances of the 'rotavirus' in negatively stained faecal extract of an infant with acute gastroenteritis. $\times 126,000$.

continuous at the periphery of the virions giving the impression of a continuous membrane surrounding the virus (intact diameter $60-65 \mathrm{~nm}$ ). The wheellike appearance of these viruses (see figure) prompted the suggestion that they be called 'rotaviruses' (Flewett et al., Lancet, ii, 61-63; 1974), though Davidson et al. (Lancet, i, 242-246; 1975 ) now suggest that 'duoviruses' may be more appropriate.

Any new genus eventually proposed would probably also include the morphologically identical viruses that cause epidemic diarrhoea of infant mice (EDIM) and some outbreaks of neonatal calf and pig diarrhoea, the simian SA11 virus and the $O$ agent isolated from gut washings in sheep and cattle. Complement fixation and immunofluorescent tests show that the human virus, the EDIM virus, and the Nebraska calf diarrhoea virus are also related antigenically. Immune electron microscopy revealed that human convalescent sera reacted with the human and calf viruses, both the intact particles and those which had lost their outer capsid layer, whereas calf antibody reacted only with the inner capsid layer of the human particles. Thus it seems that the inner capsid antigens are the common group antigens detected by complement fixation and immunofluorescent tests and the outer capsid antigens are probably typespecific. This view is reinforced by the finding that the human viruses do not infect calves.

The calf, pig and EDIM virus have been grown in tissue culture and lately the human virus has been shown to replicate in foetal intestine organ culture. Moreover immunofluorescent studies showed that the virus-specific antigen was localised in the epithelial lining of the villi (Wyatt et al., J. infect. Dis., 130, 523-528; 1974) which supports the earlier duodenal biopsy findings of Bishop et al. (1973). In the future the development of techniques by which human rotaviruses may be propagated to high titres in all cultures may eventually lead to development of a vaccine. An orally administered attenuated vaccine effective against the calf diarrhoea virus has already been prepared.

No other viruses have conclusively been shown to cause gastroenteritis in older children and adults. Parvoviruses (22 nm, DNA viruses) may cause bovine enteritis and similar viruses have been found by immune electron microscopy in stools from patients with gastroenteritis. They seem, however, to be found as easily in patients without gastroenteritis. Slightly larger $(27 \mathrm{~nm})$ picorna-like viruses have been detected in faecal extracts from an outbreak of gastroenteritis in Norwalk, Ohio. Transmission of infection and the detection of antibody to the Norwalk agent in infected people by immune electron microscopy have also been achieved. But the similarity of such small isometric particles to bacteriophages necessitates extreme caution in the interpretation of the findings in the electron microscope, and the specificity of virus-agglutinating reactions in immune electron microscopy studies has yet to be confirmed. In pigs and calves coronaviruses are also an important cause of enteritis though no cases have yet been reported implicating this group with human gastroenteritis.

Overall, existing evidence points to rotaviruses as a most important cause 
of infantile gastroenteritis throughout the world. It is important to note, however, that to date few specimens have been examined from regions where gastroenteritis mortality rates are particularly high. The use of the electron microscope for rapid and reliable detection of rotaviruses in clinical specimens and the development of serological methods of identification will greatly facilitate further studies.

\section{Does Hex A depend on Hex B?}

\section{from a Correspondent}

THE question of the relationship of the $\mathrm{N}$-acetyl hexosaminidases is still in dispute. It has been known for six years or so that two major electrophoretic forms of hexosaminidase (A and $B$ ) occur in human tissues and also that one of these (Hex $A$ ) is deficient in patients with Tay-Sachs disease, a fatal recessive disorder of infants characterised by the accumulation of a complex lipid, the ganglioside $\mathbf{G M}_{2}$, the in vivo substrate of the enzyme, in the brain. The deficiency of both enzymes is also found in some patients -a variant which is often called Sandhoff's disease. It has generally been thought to be unlikely that two separate and independent gene loci code for the hexosaminidases, as the deficiency of both A and B in Sandhoff's disease would presumably require two separate mutations (at the locus for $A$ and for $B$ ). The deficiency of Hex B alone has never been reported and TaySachs disease is extremely rare-thus the coincidence of the two homozygous mutant genes in the same individuals would be expected to be very rare indeed. But twenty or so cases have in fact already been reported.

Biochemical and immunological evidence suggests that the enzymes are closely related, and two types of model have been proposed for their relationship. One is that Hex $\mathbf{A}$ and $\mathbf{B}$ possess similar and dissimilar subunits (for example $\mathrm{A}=\alpha \beta ; \mathrm{B}=\beta \beta$ ). The other is that $\mathbf{A}$ and $\mathbf{B}$ are essentially the same protein, one enzyme being a secondarily (possibly enzymatically) modified form of the other.

In theory one might expect to learn something from the study of somatic cell hybrids. Human-rodent hybrid cells progressively lose their human chromosomes. By analysing the hybrids in a search for the coincident presence or absence of human enzymes and chromosomes genes can be assigned to particular chromosomes. When more than one gene (situated on more than one chromosome) is required to code for a particular protein or proteins the number of independently located genes involved can be determined.

Information of this kind has been obtained for hexosaminidase but the results from different laboratories are conflioting (van Someren et al., Humangenetik, 18, 171, 1974; Lalley et al., Proc. natn. Acad. Sci. U.S.A., 71, 1569, 1974; Nguyen Van Cong et al., C.r. Acad. Sci. Paris., 278, 1761, 1974; Gilbert et al., Proc. natn. Acad. Sci. U.S.A., 72, 263, 1975). In the most recent paper on the subject Gilbert et al. claim that there are indeed two independent gene loci that code for the hexosaminidases. The gene for Hex $B$ is assigned to chromosome 5 and the gene for Hex A (in agreement with the other groups) is shown to be on the same chromosome as mannose phosphate isomerase(MPI), which has now been assigned to chromosome 15 (van Heyningen et al., Ann. Hum. Genet., 38, 295; 1975). This result differs from that obtained previously by Lalley et al. and Nguyen Van Cong et al. These authors never observed the retention of human Hex A in the absence of Hex B in the hybrid cells and suggested that Hex A requires the presence of two human genes for its expression and B requires only one of the two genes (which now seems to be on chromosome 5). These findings were consistent with both the favoured models for Hex A/Hex B relationship. The lack of observation of one particular combination is not however in itself very convincing evidence for a dependence model. The best supporting evidence would come from clones which have MPI but neither Hex A nor Hex B, predicted from this model for those clones which have chromosome 15 but not 5. Very few definite clones of this type have so far been reported. Gilbert et al. on the other hand claim to have clones of all the possible types (including Hex $A+$, Hex $B-$ ), which are consistent with their model. There are, however, some major problems that cast doubt on the interpretation of their results.

In order to avoid implicating two mutations in Sandhoff's disease, Gilbert et al. have suggested that the defect may be a mutation in a locus which controls the expression of both $A$ and $B$ or makes an activator. The first problem is that there is some evidence that certain Sandhoff patients at least make non-active antigenically cross reactive 'hexosaminidase' proteins (Srivastava and Beutler, J. biol. Chem., 249, 2054, 1974; Carroll and Robinson, Biochem. $J ., 137,217,1974)$. If the control model were right then no such protein should be synthesised. Second, another informative hexosaminidase variant has been reported in a healthy parent of a patient with Sandhoff's disease (Dreyfus et al., New Eng. J. Med., 292, 61,
1975). This individual has a deficiency of both Hex A and Hex B, as detected using an artificial substrate, and is probably heterozygous for the Sandhoff allele and one which produces kinetically abnormal $\mathbf{A}$ and $\mathbf{B}$ enzymes (which seem to hydrolyse the natural but not the artificial substrates for the enzyme). This variant strongly suggests that the enzymes must have polypeptides in common.

From the technical point of view, other workers have commented on difficulties involved in identification of the human hexosaminidase components in hybrid cells, and it may be that a problem of this kind is leading to misclassification of the enzymes.

So, the argument goes on ... .

\section{Genes, enzymes and behaviour}

from T. J. Crow

THAT heredity is a powerful determinant of behaviour was first cogently argued in Lange's book Crime as Destiny (Allen and Unwin, London, 1931). The controversy about the genetic contribution to intelligence has recently had a lively renaissance; $H$. J. Eysenck, a protagonist of the innate factor school, has also championed the view that there are important hereditary influences on the personality variables neuroticism and extraversion. In the more limited field of overt psychiatric disease the contribution of genetic factors to the major psychoses, schizophrenia and manicdepressive illness, is now generally accepted and has recently been reemphasised by studies on adopted offspring of schizophrenic patients (for example Heston, Science, 167, 249; 1970; Kety, Am. J. Psychiat., 131, 957; 1974). Evidence for genetic factors in neurotic illness (for example Shields, Monozygotic Twins; Oxford University Press, 1962) is less well-known, but perhaps equally significant.

Attempts to study the links between genes and behaviour have led to the development of animal models. In selective breeding experiments in rats Broadhurst (Nature, 184, 1517; 1959) was able to demonstrate a genetic influence determining 'emotionality', as defined by increased defaecation rates in an open field apparatus, and an independent factor of "extroversionintroversion' (J. exp. Psychol., 56, 349; 1958).

Bovet and his colleagues (Science, 163, 139; 1969) demonstrated very striking differences between strains, and consistencies within strain, in the rates, and temporal patterns, of aquisition of a conditioned avoidance response in mice. Such experiments invite specula- 